\title{
Salamandra robotica II: an amphibious robot to study salamander-like swimming and walking gaits
}

\author{
Alessandro Crespi, Konstantinos Karakasiliotis, André Guignard and Auke Jan Ijspeert, Member, IEEE
}

\begin{abstract}
In this paper we present Salamandra robotica II, an amphibious salamander robot, that is able to walk and swim. The robot has four legs and an actuated spine that allow it to perform anguilliform swimming in water and walking on ground. The paper first presents the new robot hardware design, which is an improved version of Salamandra robotica $I$. We then address several questions related to body-limb coordination in robots and animals that have a sprawling posture like salamander and lizards as opposed to the erect posture of mammals (e.g., in cats and dogs). In particular, we investigate how the speed of locomotion and curvature of turning motions depend on various gait parameters such as the body-limb coordination, the type of body undulation (offset, amplitude and phase lag of body oscillations), and the frequency. Comparisons with animal data are presented, and our results show striking similarities with the gaits observed with real salamanders in particular concerning the timing of body's and limbs' movements and the relative speed of locomotion.
\end{abstract}

\section{INTRODUCTION}

O $\mathrm{NE}$ of the key characteristics of animals is their ability to efficiently move in their environments. This fundamental yet impressive capability is the result of millions of years of evolution, and its flexibility and energy efficiency are still far from being approached by robots.

Understanding animal locomotion as well as developing robots capable of good locomotion are hard problems because of the complex nonlinear interactions between the control, the body, and the (unstructured) environment. Animal studies and robotics can mutually benefit from each other to improve our understanding and control of these complex interactions: robotics can benefit by taking inspiration of principles of animal locomotion to construct more agile robots, and animal studies can use robots as scientific tools to test hypotheses and better understand animal locomotion.

This article is part of this second stream and its aim is to present our progress in developing and characterizing an amphibious robot that will be used to study salamander locomotion, and in particular its locomotor neural circuits in the spinal cord. The focus is on designing a robot with (1) the sprawling posture observed in salamanders and lizards and with (2) the ability to both swim and walk like salamanders, two aspects that have received relatively little attention so far in robotics.

The salamander, a four-legged amphibian that is able to swim and walk, is an excellent model to investigate vertebrate locomotion. It is generally considered as closely similar to

A. Crespi, K. Karakasiliotis, A. Guignard and A.J. Ijspeert are with the Biorobotics Laboratory, Faculty of Engineering, Ecole Polytechnique Fédérale de Lausanne, CH-1015 Lausanne, Switzerland.

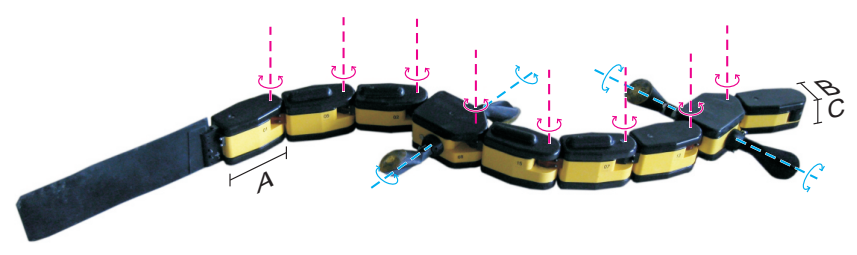

Fig. 1. The Salamandra robotica II amphibious robot. The body and limb rotation axes are shown as dashed lines. Dimensions are $A=9.5 \mathrm{~cm}, B=$ $4.7 \mathrm{~cm}$ and $C=5.8 \mathrm{~cm}$.

the first vertebrates that made the transition from aquatic to terrestrial environments, and is thus a key animal in this evolution [1], [2]. It also has orders of magnitudes fewer neurons than mammals [3], [4], thus being easier to study and model from this point of view. Finally, its central nervous system has many similarities with that of the lamprey (an extensively studied primitive fish), whose swimming circuitry is well studied and modeled, therefore assisting with the understanding of the locomotion circuits of the salamander.

In previous work, we have developed neural models of the circuits underlying swimming and walking of the salamanders to test biological hypotheses related to the transition from swimming to walking during vertebrate evolution and to the mechanisms underlying gait generation and gait transitions [5], [6]. A robot, Salamandra robotica I, was designed and constructed to validate some of these hypotheses. Using a robot, as opposed to a numerical model of the body in a physical simulation, has the advantage that it offers "real" physics, which is particularly useful for locomotion that involves complex media such as water and unstructured terrain (which are notoriously hard to simulate properly).

In this article we present Salamandra robotica II (Fig. 1) a new and improved version of Salamandra robotica I, that was briefly described in the supplementary material of [6]. The novel features of the robot include more degrees of freedom, higher torques, improved electronics, a new limb design, and a passive tail. These new features lead to significantly faster swimming and walking.

The goal of this article is three-fold: (i) to describe the robot design and how the design choices were motivated by the aim of developing a useful tool to study animal locomotion, (ii) to compare its locomotion to animal data, and (iii) to address three scientific questions related to salamander-like locomotion. These questions are: (i) how do the properties of the body undulations affect the speed of locomotion during swimming and walking? (ii) which coordination between body and limbs optimizes walking speeds? and (iii) what is the effect of asymmetric body curvature on the robot's trajectory? 


\section{RELATED WORK}

Robots are being used increasingly as research tools to verify biological hypotheses or as models of biological sensorimotor systems [7]. Examples of this include lamprey locomotion [8], [9], lobster locomotion [10], cricket phonotaxis [11] and cat locomotion [12]. For a more detailed review, see [13], [14].

Besides Salamandra robotica I [6], only a few other prototypes of salamander-like robots (i.e., quadruped robots with several degrees of freedom in the spine) have been object of scientific publications:

- Robo-Salamander, a salamander-like robot with two degrees of freedom for the spine, and two for each leg, has been presented in [15]; no experiments with it have been published, and no other publications followed. It is tethered (both for power and control), and is only capable of walking.

- A salamander robot with 6 segments and an on-board FPGA-based control system has been presented in [16]. It is not amphibious and can only walk.

Some gecko-like robots (e.g., StickyBot [17]), whose morphology has similarities to the one of the salamander, have been built. They have however no flexible spine so far, they are not amphibious, and are focused on climbing.

Nereisbot [18], a robot inspired by the locomotion of centipedes and polychaete, has a flexible spine and multiple lateral appendages, and is capable of moving on unstructured grounds (e.g., sand). None of the robots listed here is capable of swimming, and none is fully autonomous (except for Nereisbot) or amphibious.

Also related are anguilliform swimming robots such as lamprey/eel robots [19], [20], [8], [9], [21], [22], [23], or lobster robots [24].

Finally there are several amphibious robots capable of moving both in water and on ground such as Ariel (an amphibious robot to locate mines, produced by iRobot) and AQUA [25], but none using anguilliform swimming and the sprawling posture found in lizards and salamanders.

\section{KINEMATICS OF SALAMANDER LOCOMOTION}

The salamander uses an anguilliform swimming gait very similar to that of the lamprey or the eel. The swimming is based on axial undulations which are propagated from head to tail along the body, with a wave number of approximately one body length [26], [27]. The limbs are folded backwards along the side of the body. While the wave number of the undulation is kept more or less constant, the speed of swimming is mainly modulated by the frequency of the waves, as in fishes [28], but no significant correlation has been shown for the amplitude [29].

On ground, the salamander uses for slow speeds a diagonalcouplets lateral sequence walk and for higher speeds a walking trot [30], [31]. In a trot gait opposite limbs are out of phase, while diagonal limbs are in phase. In a walking gait the duty factor (the ratio between stance and swing durations) is larger than 0.5 . In this article we will focus on the walking trot gait and we will call this the walking gait for short. The limbs are coordinated with the body such that a forelimb and the contralateral hindlimb are protracted during the contralateral trunk contractions. This coordination allows the salamander to increase its stride length in this sprawling gait [32], [33], as will be confirmed in this article. In most salamander species, the tail and part of the trunk remain in contact with the ground during the whole step cycle. This ground contact increases the stability of the trotting gait. However, exceptions exist for salamanders whose limbs are strong enough to lift the whole body [34].

Finally, another difference between swimming and walking in salamanders is that the frequency of the swimming movements is generally 2-3 times higher than that of the walking movements [35], [27], [26]. For a more detailed review of salamander kinematics and the underlying locomotor circuits, please read [36].

\section{ROBOT'S MECHANICS}

\section{A. Design considerations}

Both versions of our salamander-like robots, Salamandra robotica $I$ and $I I$, are a clear case of bioinspired design. As discussed in the introduction, the aim for constructing such a robot is two-fold: i) to serve as a tool for neuroscientific studies and ii) to advance robotics design for bimodal and efficient locomotion.

A step by step implementation of additional features inspired by the animal's locomotion is essential for understanding the level of design abstraction needed for achieving the above two goals as well as the role of each feature in locomotion. In this new version, apart from the hardware upgrades (i.e. motor torques and computational power) some new features were added and evaluated with respect to Salamandra robotica $I$. These are the folding limb design and flexible tail-fin. As for every bioinspired design, in this study we are interested in testing whether such a simple design (i.e. only few features inspired by the locomotion of salamanders) is capable of replicating the animal's locomotor characteristics at a suitable level.

We designed a robot that is as simple as possible while still allowing to match these characteristics. The robot is therefore constructed as a chain of hinge joints for lateral undulations in the horizontal plane, with rotational legs that allow creating periodic contact points with rotational thrust and switching between swing and stance phases (see Fig. 1). While the robot's swing phase is completely different from the animal limbs movements, the stance phase mimics the rotational thrust proposed by Barclay [37]. During swimming, the animal keeps its limbs folded close to the body. The addition of this feature in our new design enabled the evaluation of its importance and effect on swimming speed. Essentially the robot can locomote in two dimensions, like a previous model in simulation [5], but with the ability to move on ramps due to the compliant connections between each body segment.

The robot is constructed out of several identical elements of two types: body elements and limb elements (the head being a body element without the mechanical part). Using these two types of elements, different robot morphologies could 
also be constructed; for instance, an amphibious snake robot (Amphibot III) and a fish robot have been built.

\section{B. Spine}

As in the previous robot generations, the body elements are completely independent: each has its own power source, motor, motor controller and gearbox, and is individually waterproof without requiring an external envelope. A body element has one degree of freedom, and its casing is built by three main pieces: a body, and two covers (top and bottom), which are magnetically fixed to the body using four small magnets each. An O-ring placed between the cover and the body guarantees the waterproofing. All the envelopes are molded using polyurethane resin. The output axis is fixed in a connection piece made with slightly elastic polyurethane (but still very rigid, approx. 90 Shore A), which can be screwed to the next element, and contains six wires terminating in a custom connector, which allows a communication bus and the charging power to be distributed all along the robot. The $2.83 \mathrm{~W}$ DC motor (Faulhaber $1724 \mathrm{~T} 003 \mathrm{SR}$ ) has a maximum torque of $4.2 \mathrm{mN} \cdot \mathrm{m}$ and drives a gearbox with a reduction factor of 135 . The joint angle limits of the output axis are $\pm 55^{\circ}$.

Overall the robot is constructed as a chain of body elements with 2 limb elements in the chain (Fig. 1). The total length is $110 \mathrm{~cm}$. The axes of rotation of all body elements are aligned, which means that the robot can only make lateral undulations. Our hypothesis is that lateral undulations are far more important for sprawling locomotion than vertical. In biological studies of sprawled animals all the focus is given on the lateral undulations of the spine while no study has yet discussed vertical movements. From observation, however, the animal's spine can flex on the sagittal plane, a feature that was added to our robot by using flexible connection pieces. The flexibility in the connections leads to some (passive) vertical bending, which is useful when the robot has to move over small edges between two planes (e.g., when passing from a ramp to a horizontal ground). A passive tail fin can be added to the last body element for improving thrust and hence the swimming speed. In this article, the fin is made of thin layers of plastic. Its length $(25 \mathrm{~cm})$ and compliance were determined based on speed measurements and visual characterization (which will not be discussed in the context of this paper). To achieve decreasing stiffness from the front to the back of the fin, two layers of different length of plastic were used.

\section{Limbs}

Limb elements feature three actuated joints, one of which is mechanically identical to that of the body elements, whereas the other two are independent limb joints, capable of continuous rotation. Limb motors are the same used for body joints, and drive a gearbox with a reduction factor of 90 . A limb element has therefore three degrees of freedom, and contains three motors and gearboxes.

The design of the robot's limbs is inspired by the way the limbs of real salamanders function. While swimming, the

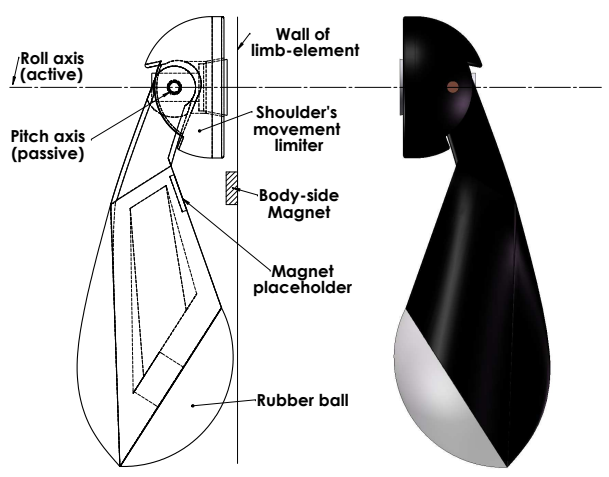

Fig. 2. The robot's limb design. The active degree of freedom rotates the limb on the roll axis. The limb can fold and unfold passively at the shoulder joint. The range of motion on the pitch axis is limited using a plastic cell. The foot is part of a rubber ball with good frictional properties.

limbs of a real salamander are tonically activated staying folded close to the body. Compared to Salamandra robotica $I$, where the limbs were always extended laterally, this functionality was added to our robot without changing the active degrees of freedom per limb.

The limb is attached to the active roll axis through a passive rotational joint which lies on the pitch axis. To limit the range of the limb's movement on the pitch axis, we covered the shoulder joint with a plastic cell which rolls along with the limb (Fig. 2). The foot of the limb is part of a rubber ball, which shows good frictional properties for a variety of surfaces. Close to the shoulder joint, at the internal side of the limb (the one closer to the body), there is a placeholder for a magnet. When the limb is folded backwards, this magnet is pulled by another magnet which is rigidly fixed on the side of the body. As a result, during swimming, when the limb is in the folded position, the force between the magnets is high enough to keep the limb at the folded position. As soon as the limb starts rotating, during walking, the distance between the magnets increases, reducing significantly their interaction. Gravity, then, extends the limb, since its center of mass is located outside the pitch axis. Note that it is mainly the drag from the water that folds the limbs backwards. The use of magnets helps to reduce any small oscillations of the freely moving limbs caused by the body movements. Fig. 3 shows a cycle of the limb's rotation and the transition from the swimming to the walking mode. The initial state (state 1) is the swimming state where the magnets reach their minimum distance. See the movie in supplementary materials.

\section{ROBOT'S ELECTRONICS}

\section{A. Body elements}

The electronics are placed on three printed circuit boards: a power board (which holds most of the components), a control board, and a connection and status display board. The connection board is mounted horizontally just below the top cover, and realizes an electrical connection between the two other boards (vertically mounted on the sides) and with the rear connector (which is connected to the robot's bus). A small transparent plastic window in the top cover leaves a zone of the connector board visible from outside, thus allowing LEDs 


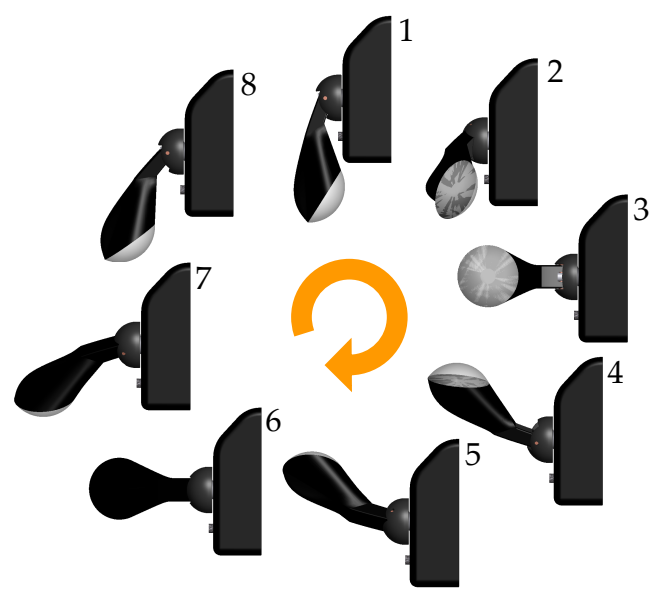

Fig. 3. Snapshots (top view) of the left side of a limb element along with the corresponding limb during one complete cycle of a limb's rotation starting from the swimming state (state 1) and switching to walking (states 2-8). Notice the body-side magnet at the bottom left of the element. The magnet on the limb is not visible in this drawing as it is embedded into the limb. State 1 : swimming posture or an instance of the swing phase of the limb. State 2: beginning of the walking state. State 3: Gravity unfolds the limb. State 5: Beginning of stance phase. State 7: End of stance and beginning of swing phase.

mounted on it to be observed by the user. A 6-wire power and data bus is shared by all the elements: two wires are used to power the battery chargers, two by the CAN bus, one to shut down the robot, and one is reserved for future use.

In this description, for simplicity, we will not distinguish on which of the printed circuits each component is located. The DC motor is controlled by a standard PD motor controller, implemented on a 8-bit PIC16 microcontroller, which drives the H-bridges. No absolute position reference is currently included in the robot, which thus require manual initialization of the zero position at startup; this is however a very easy operation.

To measure the current used by the motor (and therefore, indirectly, its torque), a $50 \mathrm{~m} \Omega$ shunt resistor is inserted between the H-bridges and the motor. The obtained voltage drop is amplified by an operational amplifier and then converted by the ADC of the microcontroller.

The circuit is powered by a $600 \mathrm{mAh} \mathrm{Li-Ion} \mathrm{rechargeable}$ battery placed near the bottom of the element, and generates the voltage required by most of the electronics $(5 \mathrm{~V})$ using a step-up converter. A switching step-down battery charger is connected to the power bus input, allowing the battery to be recharged when an external power source (typically $24 \mathrm{~V}$ ) is connected. The charging process for an empty battery is complete after about one hour. A battery protection circuit is included to avoid damages to the battery, and to prevent any dangerous conditions (e.g., high-current charging when the battery is undercharged).

An RGB LED, which can be used to display status information (e.g., battery charging status), or as a light spot for video tracking of the robot, is mounted on the top board, and is visible through a transparent window in the top cover.

To turn off the robot, a magnet is placed over a reed contact soldered on the top board of one of the elements. This allows us to turn the robot on or off without needing a waterproof mechanical switch. When the reed contact closes, it sends a shutdown signal to all the elements.

A small water detector, which proved to be very useful in rapidly detecting small leakages that can sometimes happen (e.g., if the O-rings are not perfectly greased), is placed on the lower part of the microcontroller board. When water is in contact with the sensitive surface, a bright blue LED, easily visible through the transparent window on the cover, is turned on.

A PIC18 microcontroller, connected to the CAN bus through a transceiver, acts as a bridge between the CAN bus and the motor controller (which is connected to the local $\mathrm{I}^{2} \mathrm{C}$ bus). It senses the voltage on the external power supply line, and when the power supply is connected to charge the battery, it monitors the battery protection circuit (accessed over $\mathrm{I}^{2} \mathrm{C}$ ) to display the charging state on the RGB LED, with colors depending on it. The battery information (voltage, current, current accumulator) can also be read from the CAN bus.

\section{B. Limb elements}

The electronics of limb elements are split on two boards: a motor controller board (featuring three complete PD motor controllers, including the H-bridges), and a main board, on which all the other components are mounted. The used components are the same ones used for body elements, however, some of them (the PIC16 microcontroller, the H-bridges, quadrature decoder, as well as the torque shunt and amplifier) are in triple copy, one for each motor to control.

\section{Head element}

The head element uses the same casing of body elements, but without the mechanical parts (i.e., no motor, gearbox and connection piece). It contains a controller board, based on a LPC2129 ARM7TDMI microcontroller running at $60 \mathrm{MHz}$, which is connected to the robot's CAN bus, and implements the Central Pattern Generator described in Section VI. The power board is the same used in body elements, but mounted without the motor-related components (although a complete board could also be used).

The LPC2129 communicates, using a serial line, with a small 8-bit microcontroller that which controls a nRF905 radio transceiver and implements the application layer of the radio communication protocol, also allowing the ARM microcontroller to be reprogrammed over the radio link, without any need for the head to be opened for this operation. The antenna is internal to the element and consists of a simple $\lambda / 4$ wire (where $\lambda$ is the wave number of the used frequency). The radio system uses the $868 \mathrm{MHz}$ ISM band: preliminary experiments showed that a $10 \mathrm{~mW}$ signal (the maximum power transmitted by the circuit) on this frequency band can penetrate in water up to at least $30 \mathrm{~cm}$ (the maximum tested depth). The more common $2.4 \mathrm{GHz}$ band has not been used because it is heavily absorbed by water. The maximal bandwidth that can be obtained is approximately $50 \mathrm{kbps}$, which is largely enough to send control commands and parameters to the online trajectory generator. 
A microSD slot connected to one of the SPI ports of the LPC2129 allows an optional flash memory card to be used for data storage (e.g., for logging purposes or debugging).

\section{ROBOT's CONTROL}

The robot is driven by a central pattern generator (CPG) implemented as a network of coupled nonlinear oscillators. In [6] we presented a similar network which was driving the Salamandra robotica I. The purpose of that network was to test several hypotheses concerning the mechanisms of gait transition in salamanders (swimming and walking). In this work we used a simplified version of that network ignoring, for now, the biological relevance and concentrating on the questions related to body-limb coordination as described in the introduction. However, we make use of the properties of the oscillators to control the robot's locomotion, the most important of them being the smooth transition of the output value for any change in the input parameters.

Fig. 4 shows the topology of the CPG network and the behavior of the output of each oscillator during swimming and walking. The spine (body joints) is implemented as a single chain of oscillators with bilateral couplings. There is a single oscillator for each limb. The limb oscillators are interconnected with bilateral couplings and each pair of fore and hind limbs is locally connected to the corresponding oscillator of the spine. The first oscillator is the one that drives the head, oscillators 2-5 drive the trunk, 6-8 the tail and 9-12 the limbs of the robot.

Each oscillator is implemented as an amplitude controlled phase oscillator:

$$
\begin{aligned}
& \dot{\theta}_{i}=2 \pi \nu_{i}+\sum_{j} r_{j} w_{i j} \sin \left(\theta_{j}-\theta_{i}-\phi_{i j}\right) \\
& \ddot{r}_{i}=\alpha_{i}\left(\frac{\alpha_{i}}{4}\left(R_{i}-r_{i}\right)-\dot{r}_{i}\right) \\
& x_{i}= \begin{cases}X_{i}+r_{i} \cos \left(\theta_{i}\right), & 1 \leq i \leq 8 \\
f\left(\theta_{i}\right), & 9 \leq i \leq 12\end{cases}
\end{aligned}
$$

where $\theta_{i}$ and $r_{i}$ are the state variables representing the phase and the amplitude of oscillator $i, \nu_{i}$ and $R_{i}$ determine its intrinsic frequency and amplitude, and $\alpha_{i}$ is a positive constant. Couplings between oscillators are defined by the weights $w_{i j}$ and phase biases $\phi_{i j}$. The $x_{i}$, represents the output of the oscillator after the application of an offset $X$, used to steer the robot. Note that for the experiments presented in this paper, $X_{i}=X, R_{i}=R$ and $\nu_{i}=\nu$, which means that the values of the offset, amplitude and frequency of the body's oscillations are the same for all segments.

Each output $x_{i}$ drives the corresponding spinal and limb joints of the robot, and is sent as a set point to PD controllers for each motor. Since the limbs are used in continuous rotation mode (and not in oscillatory mode), $x_{i}$ monotonically increases with $\theta_{i}$ through a piece-wise linear function $f\left(\theta_{i}\right)$ (eq. 2). That function is used as a mask applied on the phase of the corresponding oscillator and allows adjusting the stance and swinging durations separately. In this article, the ratio between swing and stance phase (i.e., the duty ratio) during
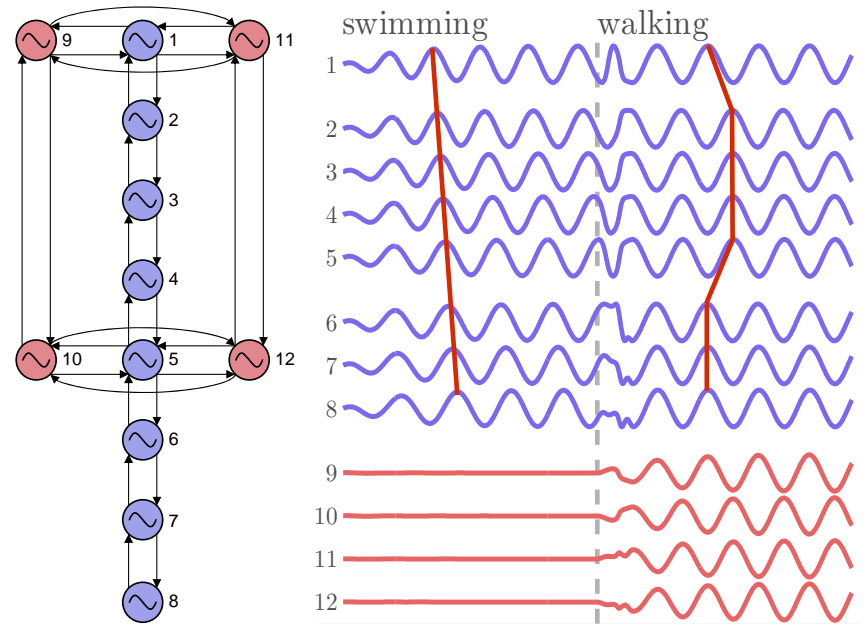

Fig. 4. On the left: The CPG network that drives the robot. Oscillators 1-8 drive the joints of the spine and 9-12 drive the limbs. On the right: Output of each oscillator for the two modes of locomotion, first swimming and then walking. Observe the traveling wave during swimming and the standing wave during walking.

walking is set to $d_{r}=50 \%$ (similarly to salamander trotting data [30]).

$$
\begin{aligned}
f\left(\theta_{i}\right) & = \begin{cases}\theta_{T G}+\left(\theta_{i}-\theta_{T G}\right) S_{f}, & \theta_{i} \leq \theta_{T G} \\
\theta_{T G}+\left(\theta_{i}-\theta_{T G}\right) S_{s}, & \theta_{i} \leq \theta_{T G i n} \\
\theta_{L G}+\left(\theta_{i}-\theta_{T G i n}\right) S_{f}, & \text { else }\end{cases} \\
S_{f} & =\frac{2 \pi-\left(\theta_{L G}-\theta_{T G}\right)}{\left(1-d_{r}\right) 2 \pi} \\
S_{s} & =\frac{\theta_{L G}-\theta_{T G}}{d_{r} 2 \pi} \\
\theta_{T G i n} & =\theta_{T G}-d_{r} 2 \pi
\end{aligned}
$$

where $\theta_{T G}$ and $\theta_{L G}$ are the angles of the limb at the start and the end of the stance phase, $\theta_{i}$ the phase of the $i^{\text {th }}$ limb oscillator translated in the interval $[-\pi, \pi]$ and $S_{f}, S_{s}, \theta_{\text {TGin }}$ some intermediate variables.

The values of the network parameters for both swimming and walking as used during the experiments are given in Table I. Note that, in Table I, $N$ denotes the number of body segments and $k$ the number of waves traveling along the body during one cycle (i.e., the inverse of the wavelength). For instance $k=1.0$ and $k=0.5$ mean respectively that a complete wave and half-a-wave travel from head to tail. $k=0$ means a standing wave. Note that for the experiments that will follow the variable $k$ is calculated only for the active degrees of freedom and it does not take into account the passive tail.

\section{CHARACTERIZATION OF THE BASIC GAITS}

In this section we characterize the performance of the robot for the two basic gaits, walking and swimming. As mentioned in the introduction, the characterization of walking and swimming is here aiming at addressing three main questions: (i) how do the properties of the body undulations affect the speed of locomotion during swimming and walking, (ii) which coordination between body and limbs optimizes walking speeds, and (iii) what is the effect of asymmetric body curvature on the robot's trajectory. 
TABLE I

NETWORK PARAMETERS

\begin{tabular}{|l|c|c|l|}
\hline parameter & walking & swimming & oscillator index \\
\hline$w_{i, i+1}$ & 20 & 20 & $i \in\{1, \ldots, 7\}$ \\
\hline$w_{i, i+2}$ & 20 & 20 & $i \in\{9,10\}$ \\
\hline$w_{i, i+1}$ & 10 & 10 & $i \in\{9,11\}$ \\
\hline$w_{i, j}, w_{i+2, j}$ & 10 & 0 & $i \in(9,10), j \in(1,5)$ \\
\hline$w_{j i}$ & $w_{i j}$ & $w_{i j}$ & $\forall i, j$ \\
\hline$\phi_{i, i+1}$ & 0 & $2 \pi k / N$ & $i \in\{1, \ldots, 7\}$ \\
\hline$\phi_{i, i+2}$ & $\pi$ & $\pi$ & $i \in\{9,10\}$ \\
\hline$\phi_{i, i+1}$ & $\pi$ & $\pi$ & $i \in\{9,11\}$ \\
\hline$\phi_{i, j}, \phi_{i+2, j}$ & 0 & 0 & $i \in(9,10), j \in(1,5)$ \\
$\phi_{i+2, j}$ & $-\phi_{i j}$ & $-\phi_{i j}$ & $\forall i, j$ \\
\hline$\phi_{j i}$ & 2 & 2 & $\forall i$ \\
\hline$\alpha_{i}$ & 9 & 9 & \\
\hline$N$ & 0 & $\neq 0$ & \\
\hline$k$ & 0.5 & - & \\
\hline$d_{r}$ & $-5 \pi / 6$ & - & \\
\hline$\theta_{T G}$ & $-\pi / 6$ & - & \\
\hline$\theta_{L G}$ & \multicolumn{3}{|l}{} \\
\hline
\end{tabular}

\section{A. Methods}

We carried out two sets of experiments: a set of experiments to characterize the gaits and the speed during straight locomotion while walking and swimming (experiments $\mathrm{A}, \mathrm{B}$, and D), and a set of experiments to investigate turning maneuvers during walking and swimming (experiments $\mathrm{C}$ and $\mathrm{E}$ ). Finally we performed experiments to compare swimming speeds with and without the flexible fin and with and without the folding limbs (Experiment F). The straight locomotion experiments are done by keeping the offset $X$ to 0.0 (eq. 1c), while the turning experiments are done by setting $X$ to different values.

To measure the speed and curvature of the robot trajectory we used two cameras placed above a $6 \mathrm{~m} \times 1.5 \mathrm{~m}$ pool able to detect and record the positions of LEDs at a rate of 15 fps using custom software. The LEDs used during these experiments were the ones embedded in each element of the robot (see Section IV). Therefore, each element was recorded as a single point. Two additional LEDs placed on the tail-fin of the robot were used to track its total length and the deformation of the flexible fin. During walking the pool was covered by wooden plates forming a corridor, while during swimming the water level was kept constant at $15 \mathrm{~cm}$ and the robot was swimming just below the surface.

Speed was measured by recording the time needed for the robot to cover a given distance while keeping its trajectory in a straight line ${ }^{1}$. During each experiment the robot covered a distance of $5 \mathrm{~m}$. The time measurement was initiated after the first $2 \mathrm{~m}$ and stopped after the line of $5 \mathrm{~m}$. The speed was therefore calculated as the distance to travel $3 \mathrm{~m}$ over the measured time. The initial $2 \mathrm{~m}$ are sufficient to ensure that the robot reaches its steady state (i.e., the CPG has converged

\footnotetext{
${ }^{1}$ To ensure that the robot does not drift away from the straight line in the middle of the pool or the wooden plates when measuring forward speed, a closed loop control loop based on visual tracking was used. The control loop modulates the offset $X$, i.e., the average curvature of the body that is used for steering, in order to keep the front girdle close to the centerline. This control loop was very useful to perform systematic experiments and only minimally affected the offset $X$ which stayed very close to zero in average. Indeed the mean value of this offset was less than $1^{\circ}$ for most of the experiments, while the maximum mean offset was around $2^{\circ}$. These values can be considered insignificant compared to the range of amplitude of oscillations used in the experiments. Note that this control loop was only used for the experiments involving straight locomotion and not during the turning experiments where $X$ was set to a constant value.
}

to its limit cycle and the robot has reached a constant speed of locomotion). We performed three runs for each parameter set.

For the experiments investigating turning behavior, we measured the curvature of the robot's trajectory by fitting a circle to the path made by the robot's geometric center of mass using the least squares method. The curvature of the path is then defined as the inverse of the radius $\left(1 / R_{f i t}\right)$ of the circle that best describes the trajectory's curve. For the swimming experiments, the curvature was only recorded after $5 \mathrm{~s}$ of straight swimming in order to ensure that the robot had reached steady-state speed. To get statistics over the curvature we let the robot run until a full circle was completed or until a pool's border was reached. Then we evaluated the curvature on three different portions of the final trajectory of the robot's CoM.

The results of the experiments are compared to biological data from real salamander kinematics that come either from the literature or from recent X-ray recordings performed together with Nadja Schilling (University of Veterinary Medicine Hannover, Foundation and Institute of Systematic Zoology and Evolutionary Biology), Martin Fischer (University of Jena) and Jean-Marie Cabelguen (University of Bordeaux). The methods and detailed analysis of those recordings are out of the scope of this article and will be described in a future article.

\section{B. Walking}

1) Experiment A, forward walking: In walking, we use four basic parameters to alter the gait's behavior. These are the amplitude $(R)$ and frequency $(\nu)$ of the body wave, the bodylimbs phase lag $\left(\phi_{B L}\right)$ and the steering offset $(X)$. For the characterization of the forward walking, we fix $\phi_{B L}=0$ and $X=0$ (except for the negligible adjustments due to visual tracking described in footnote 1) and we systematically evaluate the robot's speed for different amplitudes and frequencies. When $\phi_{B L}=0$ the switching between stance and swing phase coincides with the maximum bending of the trunk. We will explore the influence of $\phi_{B L}$ in experiment B.

Fig. 5(a) shows the measured speed for $0^{\circ} \leq R \leq 25^{\circ}$ with a step of $5^{\circ}$ ( $x$-axis) and $0.4 \mathrm{~Hz} \leq \nu \leq 1.2 \mathrm{~Hz}$ with a step of $0.2 \mathrm{~Hz}$ (different curves). On top of the plot, for each instance of $R$, there is an example of the robot's posture when the body wave is at its crest.

Both the body wave amplitude and frequency have a clear effect on the robot's speed. Speed increases with the increase of amplitude and frequency, but both of them seem to converge to a maximum value. For the amplitude, for instance, at $1.2 \mathrm{~Hz}$, as its value increases, the best speed has been already reached at $20^{\circ}$. Although, for the other frequencies the maximum speed is found at the maximum value of amplitude, it is clear that the speed rate decreases. Note that for the safety of the mechanics of the robot we did not test amplitude values higher than $25^{\circ}$. For the frequency, similarly, as its value increases, the speed rate decreases. The maximum value of frequency was limited by the ability of the motors to properly follow the desired kinematics.

The overall best speed of $0.42 \mathrm{~m} / \mathrm{s}$ was found at $R=20^{\circ}$ and $\nu=1.2 \mathrm{~Hz}$. Snapshots of one cycle of locomotion for the 


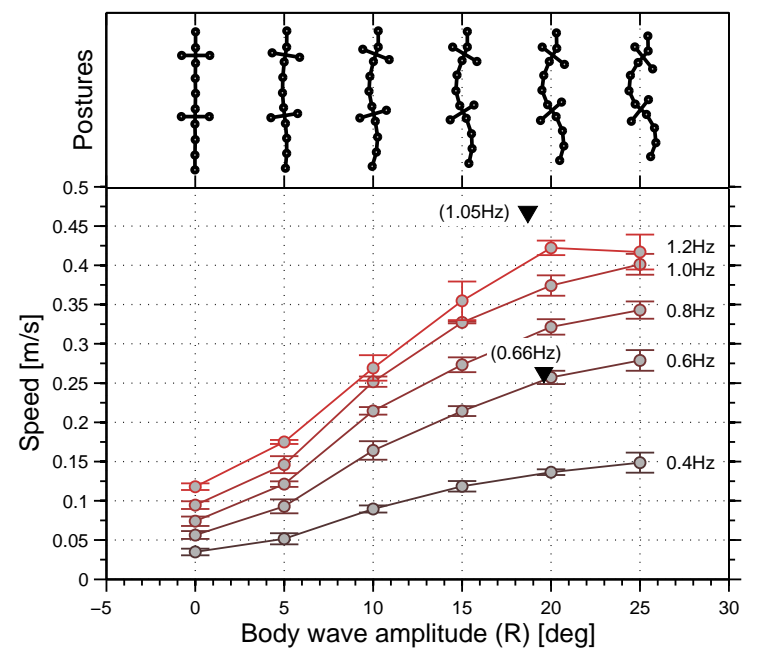

(a)

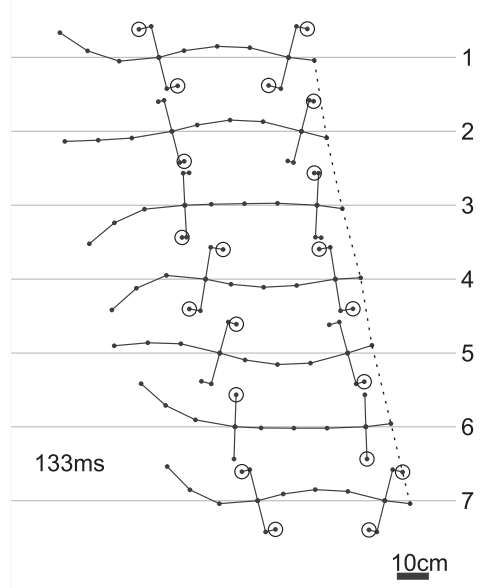

(b)

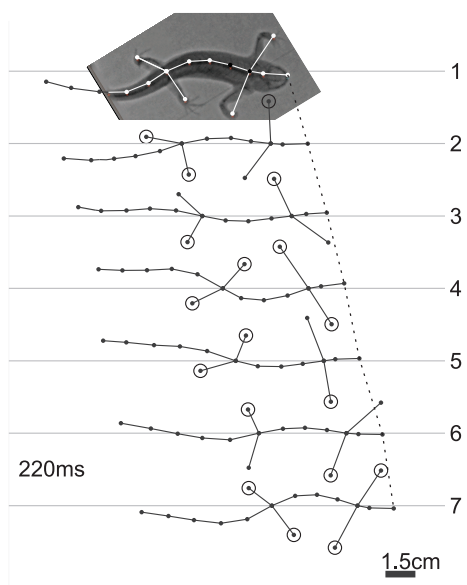

(c)

Fig. 5. Experiment A. (a) The plots with circular markers show the robot's speed as a function of the body wave's amplitude $(R)$ and frequency $(\nu)$ when $\phi_{B L}=0$ and $X=0$. The error bars correspond to the standard deviation of the different runs of the same set of parameters. On top of the plot, for each instance of $R$, there is an example of the robot's posture when the body's wave is at its crest. For comparison, two example data points, which correspond to data from real salamanders, are shown with triangular markers. The corresponding value of frequency is shown in parenthesis. Note that the speed data of the salamanders have been scaled up based on the SVL of the robot (see text). (b) Snapshots of the robot during one cycle of locomotion for the best obtained speed. (c) Snapshots of a real salamander during one cycle of walking. The stance phase of each foot is denoted by a circle. The time between each snapshot is given at the bottom left while the scale of distance is given at the bottom right.

best speed are shown in Fig. 5(b). For comparison, similar snapshots of a real salamander are presented in Fig. 5(c). Notice the similarities of the kinematics between the robot and the real salamander. The spine is oscillating using a standing wave with nodes at the two girdles, while the footsteps have very similar timings. Real salamanders, when walking at around $1.05( \pm 0.135) \mathrm{Hz}$ use an amplitude of body oscillation of $18.7^{\circ}\left( \pm 2.9^{\circ}\right)$ and their speed is $0.78( \pm 0.031) \mathrm{SVL} / \mathrm{s}$ [30] (Snout-Vent-Length; the distance between the snout and the vent). This speed would be $0.47( \pm 0.018) \mathrm{m} / \mathrm{s}$ when rescaled to the size of the robot. ${ }^{2}$

The robot, for similar kinematic variables $(\nu=1.2 \mathrm{~Hz}$ and $R=20^{\circ}$ ), has a speed of $0.42 \mathrm{~m} / \mathrm{s}$. At lower frequencies $(0.66( \pm 0.135) \mathrm{Hz})$ salamanders use an amplitude of body oscillation of $19.6^{\circ}\left( \pm 3.4^{\circ}\right)$ and their speed is 0.264 $( \pm 0.01) \mathrm{m} / \mathrm{s}$ [30]. The robot for $\nu=0.6 \mathrm{~Hz}$ and $R=20^{\circ}$ has a speed of $0.26 \mathrm{~cm} / \mathrm{s}$ (Fig. 5(a)). This comparison can be made also in Fig. 5(a) where the animal data are shown with triangular markers. The above two examples show striking kinematic correspondence between the robot and the animal during walking.

2) Experiment B, Body-Limbs coordination: By body-limbs coordination we refer to the phase relationship between the rotation of the limbs and the phase of the body's wave. For instance, as mentioned before, when the rotation of the limbs and the wave of the body are in phase $\left(\phi_{B L}=0\right)$, the switch between stance and swing phases coincides with the maximum bending of the trunk.

To illustrate the importance of this parameter, we systematically tested its effect on speed for the forward walking. For given values of amplitude and frequency, $R=15^{\circ}$ and

\footnotetext{
${ }^{2}$ The animal's speed is scaled to the robot's units using the relative SnoutVent-Lengths of the two. The vent of the robot is approximated to be at the back side of the hind-limbs' body segment. The robot's SVL then is $60 \mathrm{~cm}$.
}

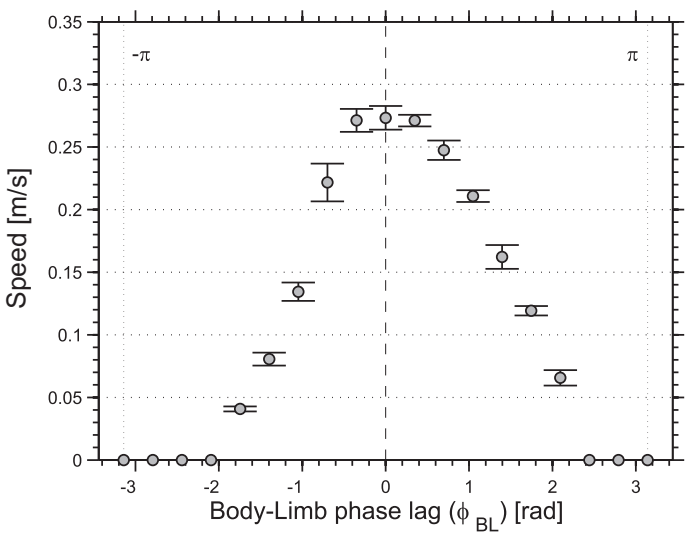

Fig. 6. Experiment B. Speed as a function of body-limbs phase lag, $\phi_{B L}$, for $R=15^{\circ}$ and $\nu=0.8 \mathrm{~Hz}$. The dashed vertical line at 0 shows the body-limbs phase lags observed in real salamanders.

$\nu=0.8 \mathrm{~Hz}$, we varied the $-\pi \leq \phi_{B L} \leq \pi$ with a step of $\pi / 9$ and, using the same setup as for forward walking, we measured the robot's speed (Fig. 6). Again, we performed three runs for each set of parameters. As the phase difference $\phi_{B L}$ moves away from 0 , there is a rapid decrease in performance with the smallest values of speed around $\pi$. Around $\pi$, the robot was either oscillating in place or was slightly moving backwards, but on a very unstable trajectory. The best performance is obtained at $\phi_{B L}=0$ which is, not surprisingly, the same timing observed in real salamanders (Fig. 5(c)); this explains our initial choice of $\phi_{B L}$ for the forward walking experiments, which was discussed earlier.

To explain the effect of body-limbs phase lag on speed, we compare the robot's kinematics for two different values of $\phi_{B L}:$ (1) one that gives the best performance, $\phi_{B L}=0$ and (2) one which gives significantly decreased speed, $\phi_{B L}=$ $5 \pi / 9$. In Fig. 7(a) and (b) we show seven snapshots of the robot for one complete locomotion cycle, with $\phi_{B L}=0$ and 


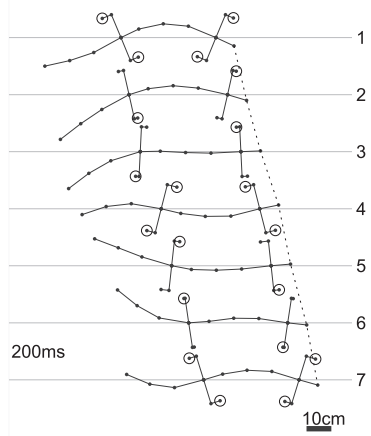

(a)

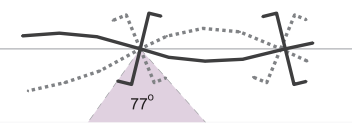

(c)

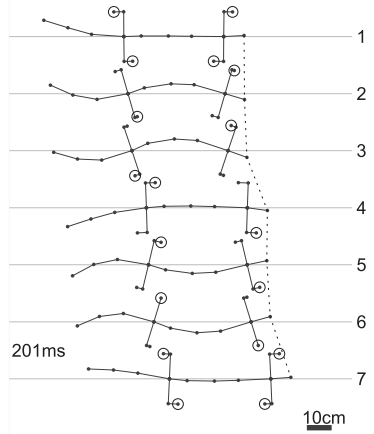

(b)

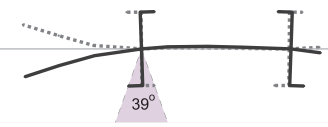

(d)
Fig. 7. Experiment B. Snapshots of the salamander robot during one period of walking with $R=15^{\circ}, \nu=0.8 \mathrm{~Hz}$ and, (a) and (c) $\phi_{B L}=0$, (b) and (d) $\phi_{B L}=5 \pi / 9$. In (a) and (b), a circle on the end point of each limb denotes that the limb is in stance phase. The time-step between the snapshots is shown at bottom left. In (c) and (d), we show two snapshots of the robot at the start (gray) and at the middle (black) of the cycle. The angles between the dashed lines show the resulting range of the limb during the stance phase.

$\phi_{B L}=5 \pi / 9$ respectively. The amplitude and frequency of the body wave are the same for both runs, with $R=15^{\circ}$ and $\nu=$ $0.8 \mathrm{~Hz}$. The circles on the feet of the robot indicate that the corresponding limb is in stance phase. In the first place, when body and limbs are in phase, the initiation of the locomotion cycle coincides with the maximum bending of the trunk (frame 1 in Fig. 7(a)). On the other hand, when $\phi_{B L}=5 \pi / 9$, the initiation of the locomotion cycle finds the trunk in a straight posture (frame 1 in Fig. 7(b)). Throughout the next two frames, as the trunk starts to bend, it counteracts the thrust provided by the limbs. Later, as the trunk unfolds, the limbs are near the end of their stance phase, which means that they can still provide some leverage for the trunk to produce thrust. This explains the sudden displacement of the robot in the middle of the cycle. But since the foot has rotated backwards, the resulting thrust is decreased. The snapshots in Fig. 7(c) and (d) summarize the above analysis. They correspond to the postures of the robot at the start (gray) and the end (black) of the stance phase (half cycle). Here, it is clear that the range, in which the limbs are active, is much wider when $\phi_{B L}=0\left(77^{\circ}\right)$ than when $\phi_{B L}=5 \pi / 9\left(39^{\circ}\right)$. Of course, supposing that the feet do not slip, the wider the range, the longer the stride length. Note that, here, we prefer to describe the range of limb activity as an angle. In this way, we emphasize the fact that the increased stride length is a result of the well timed rotation of the hip (caused by the trunk bending).

3) Experiment $C$, turning on ground: Turning is generated by a non-zero offset $(X)$ in the wave of the body. The sign of this offset controls the direction of the turn, while its value controls the curvature. Since the robot and its gaits are symmetric, it is expected that the turning behavior is the same on both sides. This means that the trajectories for left and right turns should have the same curvature.

Varying the offset and the amplitude of the body's wave, $5^{\circ} \leq X \leq 20^{\circ}$ and $0^{\circ} \leq R \leq 15^{\circ}$ with a step of $5^{\circ}$, we

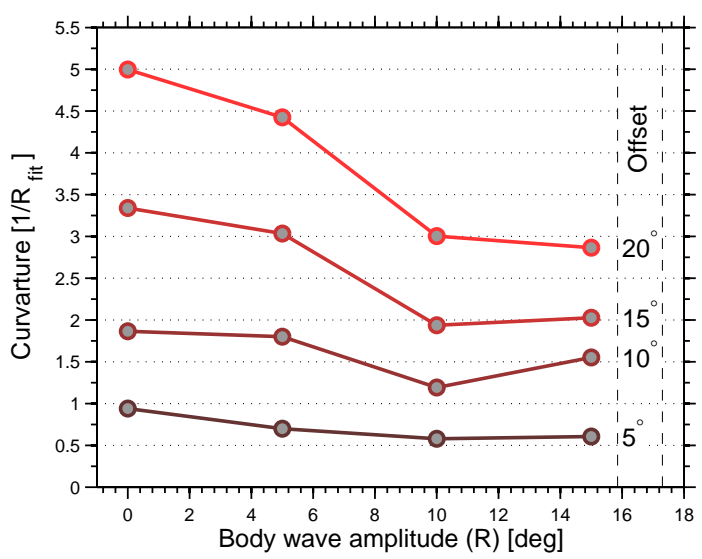

Fig. 8. Experiment C. Curvature of the robot's trajectory as a function of the body's wave amplitude, $R$ ( $x$-axis), and offset, $X$ (different curves), for a fixed frequency of $\nu=0.8 \mathrm{~Hz}$.

measured the curvature of the resulting turn (Fig. 8). We used a fixed value for the frequency, $\nu=0.8 \mathrm{~Hz}$, as we expect that the results should be qualitatively similar for other frequencies. The limits for both parameters were set to ensure the robot's safety and avoid high torques and internal collisions.

Fig. 8 shows the resulting curvature of the robot's trajectory as a function of the body's wave amplitude ( $x$-axis) and offset (different curves). Increasing the offset of the body's wave curves the robot at one side. Therefore it is of no surprise that the curvature is increasing using higher offsets. However, it is interesting to see that the amplitude has a negative impact on the trajectory's curvature. In Fig. 9 we show the trajectories of the robot for the two extreme values of amplitude $R=0^{\circ}$ and $R=15^{\circ}$. The different curves in each plot correspond to different values of offset. The heavy gray lines are the trajectories of the robot's geometric center of mass and the fitted circle is shown with a green dashed line. As shown in Fig. 8, the more curved trajectories correspond to higher offsets. The effect of the amplitude is becoming more important when the offset increases. Its effect, however is inversely proportional to the resulting curvature. The effects of offset and amplitude can be seen in the example trajectories shown in Fig. 9. In Fig. 9(a) the amplitude is lower than in Fig. 9(b). Four different values of offset are generating four different trajectories (thick lines), with the more curved ones being the result of higher values of offset.

Turning on ground has not yet been quantitatively studied in real salamanders. From video recordings, one can qualitatively observe that curvature of the trunk is higher on one side (data not shown), but this has not been properly quantified. Similarly, the contributions of the limbs to turning have not been quantified. During the experiments with the robot, slipping of the feet on the ground has been observed for higher turning curvatures. This could suggest that the segmented limbs of salamanders can regulate their posture in order to maintain initial feet positions. The usefulness of a knee joint during turning has been discussed before in simulation in [38].

\section{Swimming}

1) Experiment D, forward swimming: We performed systematic tests for three parameters of the swimming gait, the 


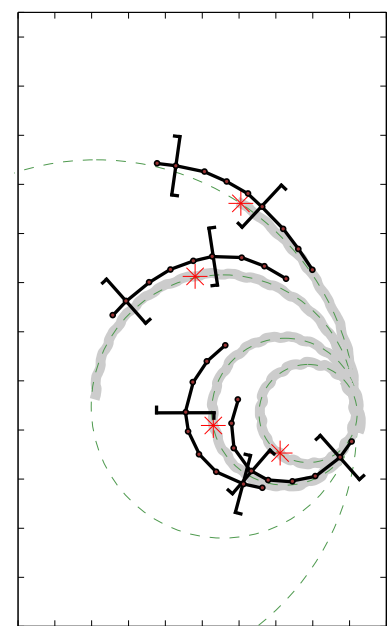

(a)

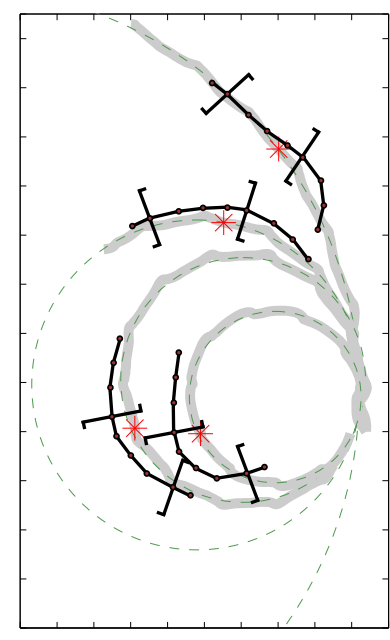

(b)
Fig. 9. Experiment C. Trajectories of the salamander robot's geometric center of mass (thick lines) for different values of body's wave offset (different curves in the same plot) and for the two extremes of amplitude (a) $R=0^{\circ}$ and (b) $R=15^{\circ}$. The fitted circle for each trajectory is shown with a dashed green line. For each trajectory, there is one instance of the robot and its geometric center of mass (red star).

amplitude $10^{\circ} \leq R \leq 30^{\circ}$ with a step of $5^{\circ}$, frequency $0.4 \mathrm{~Hz} \leq \nu \leq 1.0 \mathrm{~Hz}$ with a step of $0.2 \mathrm{~Hz}$ and wave number $0.5 \leq k \leq 1.5$ with a step of 0.5 , of the body's wave. Note that the limits of the parameters' space were again defined based on the safety and the limitations of the hardware. Fig. 10(a),(d) and (f) show the robot's swimming speed as a function of the amplitude and frequency of the body's wave for three different wave numbers $(k)$. The error bars correspond to the standard deviations of the three different runs for each set of parameters.

Decreasing the number of waves per body length $(k)$, the speed increases. Increasing the amplitude and frequency of the body's traveling wave the speed, also, increases. Increasing the wave's amplitude, for any values of frequency and wave number, the resulting speed tends to reach a crest. For all wave numbers, the maximum speed is obtained when the amplitude is $R=30^{\circ}$ and the frequency is $\nu=1.0 \mathrm{~Hz}$. For $k=0.5$ the maximum speed is $0.51 \mathrm{~m} / \mathrm{s}$ - which is the over all best-, $0.45 \mathrm{~m} / \mathrm{s}$ for $k=1.0$, and $0.32 \mathrm{~m} / \mathrm{s}$ for $k=1.5$.

In Fig. 10(b),(e) and (g) we show snapshots during one period of swimming for the best values of amplitude and frequency for the different wave numbers. A big circle at each plot indicates the set of parameters which give the best speed for a given wave number. From these snapshots, one can see how the wave number $(k)$ affects the posture and, eventually, the performance of the swimming gaits. In Fig. 10(c) we present snapshots of a real salamander swimming for one locomotion cycle. From a kinematics point of view, the robot shows similar behavior at each snapshot with the real salamander. Moreover, the similarity in the forward displacement of the head at each snapshot (given the similar kinematics) shows that there is similarity also in the wave efficiency (ratio of forward speed to wave speed). However, there are two main differences between the robot and the animal: i) the animal stabilizes the orientation of the anterior body and head ${ }^{3}$, i.e., the head stays more aligned with the direction of swimming, and ii) the posterior part of the tail, being active in the animal, can perform finer movements. Due to the non-constant amplitude used by the salamanders during swimming, a direct comparison with the robot is not possible. However, it is possible to compare their performance by comparing their stride length as defined, and measured for steady swimming salamanders, in [29]. The stride length represents the number of body lengths traveled within one complete swimming cycle and is calculated as $U / \nu L$, where $L$ is the length of the robot or the animal, $U$ the speed and $\nu$ the frequency of locomotion. The stride length of the robot for the best measured speed is then 0.447 while for the salamanders in [29] was $0.345( \pm 0.077)$. However, the number of waves that salamanders used in that paper was $k=1.675( \pm 0.27)$ which comes closer to $k=1.5$ used in the robot. Then, for the best speed at $k=1.5$ the stride length of the robot drops down to 0.28 which is closer to the mean value of the real salamanders. That the recorded salamanders use higher number of waves, which according to the results from the robot is not optimal for speed, could mean that during steady state swimming salamanders optimize something else than speed, for instance efficiency. Note that a direct comparison between the undulatory amplitude of the robot and the animal is not possible. No data are yet presented in literature describing angular spinal kinematics, but, rather, the amplitude of lateral displacement of the tail's tip is used. This is because real salamanders gradually increase the undulatory amplitude from head to tail. Although this could be explored with our robot in future work, here we focus on constant amplitude along the whole body.

2) Experiment E, turning while swimming: Turning in swimming is generated in the same way as in walking, that means, by varying the body's oscillation offset $(X)$. Apart from the amplitude $(R)$ and offset $(X)$, we evaluated the effect of the wave number $(k)$ on the turning curvature. Again, as for the over-ground turning, the frequency was set to $\nu=0.8 \mathrm{~Hz}$.

Fig. 11 shows the results for values of wave number (each plot) $k=0.5, k=1.0$ and $k=1.5$, for four different amplitudes (each curve) $10^{\circ} \leq R \leq 25^{\circ}$ and six values of offset ( $x$-axis) $2^{\circ} \leq X \leq 14^{\circ}$. Note that for values of offset higher than the tested ones, the head of the robot was colliding with the tail-fin. The error bars in these plots show the standard deviation of three measurements of the curvature at different time windows of the recorded trajectory.

From the previous results we can make the following interesting observations: $i)$ increasing the offset $(X)$ the turning curvature increases almost linearly, ii) the amplitude $(R)$ does not have a significant effect on the turning curvature, or this effect is small, and iii) increasing the wave number $(k)$ the turning curvature tends to decrease, but the effect is not strong. So, interestingly, the only parameter that seems to have a strong effect on the turning curvature during swimming is the offset of the body's oscillation.

Turning has not been quantitatively studied yet in steady

\footnotetext{
${ }^{3}$ In the robot, this can be obtained by having a gradient of amplitude of oscillation from head to tail.
} 


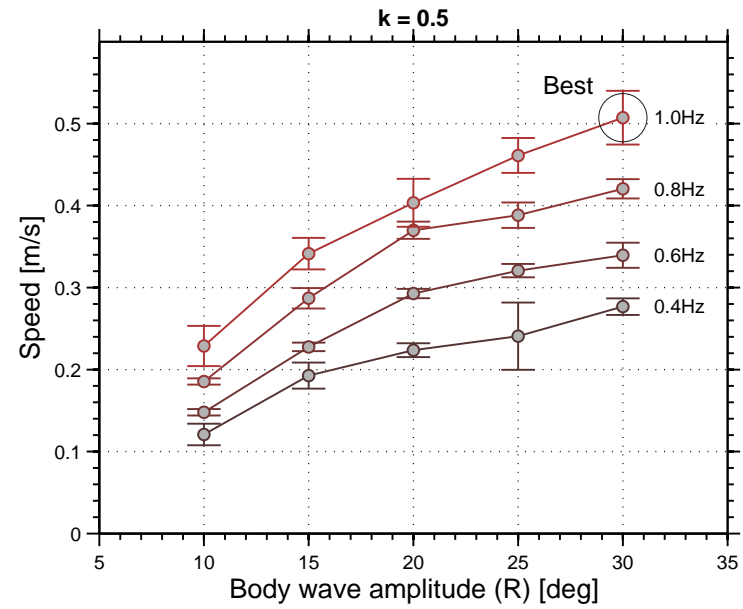

(a)

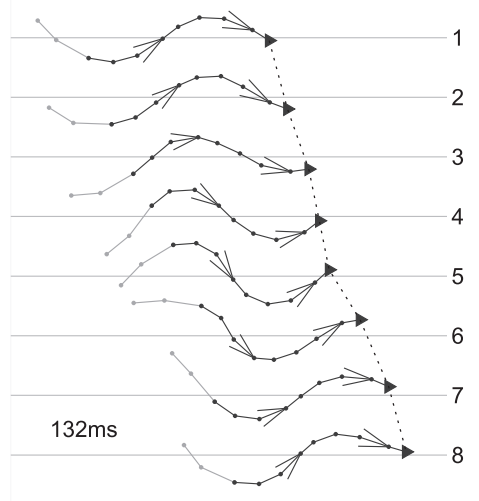

$10 \mathrm{~cm}$

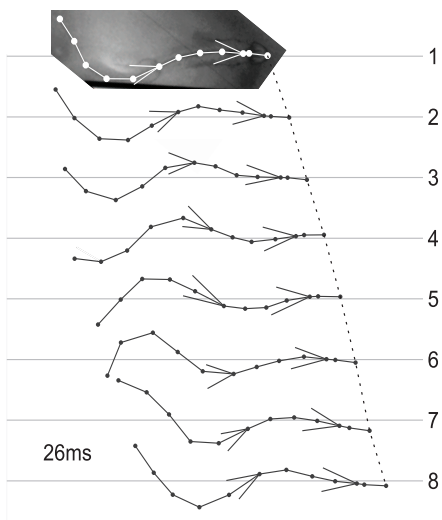

$1.5 \mathrm{~cm}$

(b)

(c)

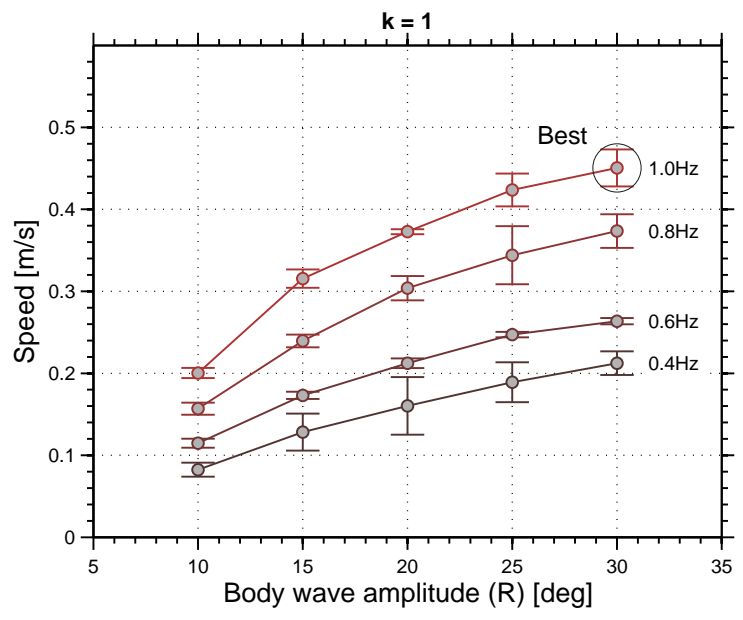

(d)

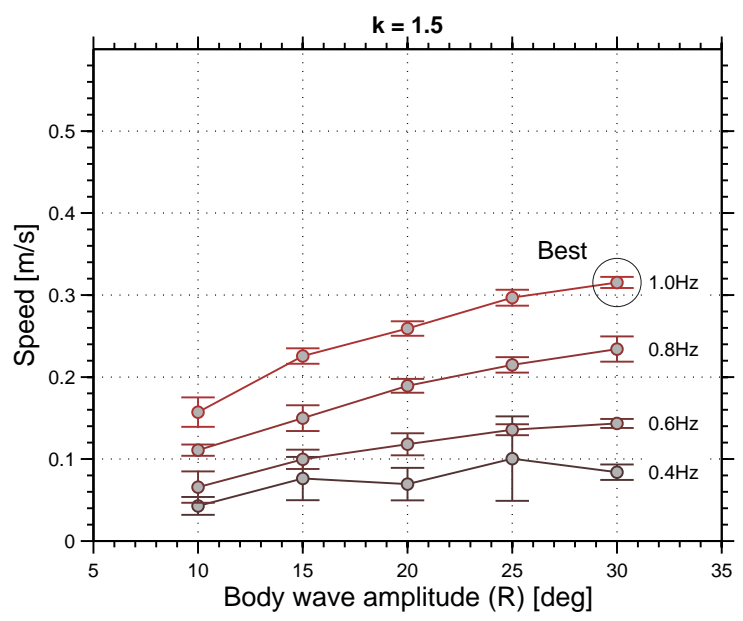

(f)

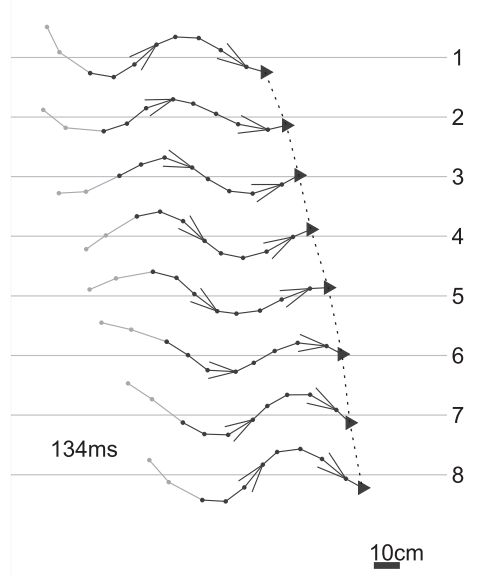

(e)

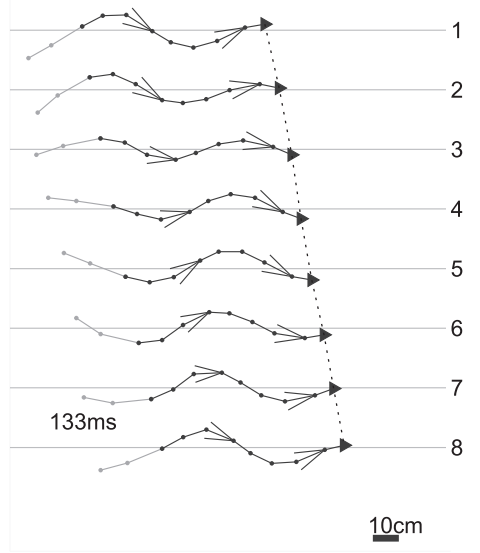

(g)

Fig. 10. Experiment D. The robot's swimming speed as a function of the amplitude $(R)$ and frequency $(\nu)$ of the body's wave and for (a) $k=0.5$, (d) $k=1.0$ and (f) $k=1.5$ waves per body length (wave number). On the right side: snapshots during one period of swimming for the best obtained performance when the wave number is (c) $k=0.5$, (e) $k=1.0$ and (g) $k=1.5$. For each sub-figure with snapshots, the filled triangles denote the head of the robot, the gray part represents the tail-fin and the time-step between consecutive snapshots is noted on the bottom-left corner. Snapshots of a real salamander swimming are shown in (c) for comparison with the best gait obtained with the robot. A big circle at each plot indicates the set of parameters which give the best speed for a given wave number. 
state swimming for salamanders. However, some studies on their escape responses [39] and video footage seem to indicate that steering during swimming is mainly achieved through stronger contractions (higher bending) on one side of the salamander's spine.

3) Experiment $F$, effects of passive tail and folding limbs on swimming speed: As for a last experiment, and a comparison of the design improvements between Salamandra robotica I and $I I$, we recorded the performance of the new robot in two different configurations than the one used for the rest of the experiments: i) without the passive tail-fin and ii) with extended limbs (i.e. by preventing them from folding). In particular, with the new robot we could identify that, in addition to the higher torques, the increase of swimming speed is mainly due to the fin (i.e., the addition of passive DOFs), followed by the more profiled limbs, and finally the additional actuated DOFs. Blocking the folding of the limbs (i.e., keeping them extended as in Salamandra robotica I) produces a speed decrease of $39 \%$, and removing the tail (with foldable limbs) produces a speed decrease of $63 \%$ compared to swimming with folded limbs and with the fin (data not shown).

\section{DISCUSSION AND CONCLUSION}

This work intended to address, on one hand, the design considerations of a new salamander-like robot and its walking and swimming performance compared to real salamanders, and on the other hand, basic questions related to salamander-like locomotion: (i) how do the properties of the body undulations affect the speed of locomotion during swimming and walking, (ii) which coordination between body and limbs optimizes walking speeds, and (iii) what is the effect of asymmetric body curvature on the robot's trajectory? We will first discuss these questions, and then come back to the robot design.

The answer to the first question is that, as could be expected, properties of the body undulation strongly affect locomotion, in particular locomotion speed. First of all, without undulation (i.e., when the amplitude $R$ is set to zero), there is no forward swimming and forward walking is much slower than with optimal amplitude parameters. Second, there is a monotonic increase of speed with the frequency, i.e., increasing the frequency systematically increases the speed of locomotion both for walking and for swimming in the tested range. Due to torque limits, the speed will eventually drop with too high frequencies, but for the tested range, changing the frequency is a convenient way to control speed, as it would logically be expected. Increasing the amplitude also tends to increase speed up to some point. Although we did not test it to avoid mechanical damages, too large amplitudes will lead to excessive bending in the spine and eventually collisions between limbs. A good way of controlling speed is therefore to increase frequency while maintaining amplitude at a constant optimal value (e.g. $20^{\circ}$ for walking and $30^{\circ}$ for swimming). Real salamanders tend to follow the same strategy for increasing speed. Indeed, the main parameter they systematically vary when changing speed is the frequency whereas the oscillation amplitude remains more or less constant for walking [30] or varies slightly but non systematically for swimming [29]. Note that these observations that speed increases monotonically with frequency and, up to some optimal value, with amplitude are by themselves not surprising. However it is interesting and important to quantify these relationships in order to quantify how much an increase of frequency and/or amplitude affects speed. In the future, we intend to also measure the related energy costs (this requires an upgrade of the electronic hardware of the robot), and this will allow us to compare tradeoffs between speed and efficiency of locomotion for different gaits (e.g. different frequencies and amplitudes).

Concerning body-limb coordination during walking, a clear optimum was obtained when $\phi_{B L}=0.0$, which corresponds to maximally protracting (i.e., extending forward) a forelimb on one side, when the trunk is maximally bent towards the other side. This optimizes the stride length and is exactly the coordination observed in salamanders [30]. Our study also showed how important this proper coordination is between body undulation and limb movements: with a wrong coordination (e.g., $\phi_{B L}=\pi$ ), the speed of locomotion drops to zero.

Concerning turning behavior, our study has shown that changing the average curvature of the body (by setting a nonzero offset $X$ ) is an efficient way of controlling the heading of locomotion, both in water and on ground. This is one of the nice properties of having a spine with multiple DOFs that can bend laterally. The curvature of the path is proportional to the offset $X$, thus making $X$ a useful control parameter for steering. During walking it was found that a higher amplitude of the body undulation can have a negative effect on the steering ability: the robot turns less sharply when the body undulates versus turning without undulation $(R=0)$. This is probably due in part to the simplified design of the limbs that do not have enough DOFs to ensure a proper closed chain loop between the two limbs on the ground. There is no detailed kinematic study of salamander turning behavior, but qualitative observations of video recordings show that salamanders actively use their trunk for steering similarly to our experiments. See also [38] for a study of turning behavior in a neuromechanical simulation that shows that best turning during walking is obtained with a combination of trunk bending and of lateral steps (that our current robot cannot do).

Despite much simpler kinematics than the real animal (in particular fewer DOFs in the spine and in the limbs), the optimal gaits produced by the robot both for swimming and walking are strikingly similar to those of a salamander. In particular, during walking, the body-limb coordination is the same. The body oscillates using a standing wave while the footsteps follow the timing of the real salamanders.

This encourages us to continue using the robot as a tool for testing hypotheses concerning gait generation and gait transition in the salamander. Our first study [6] allowed us to show how the tetrapod locomotion controller of salamanders can be built using the primitive swimming circuit of the lamprey as a base, and to explain the switch of body undulations between traveling and standing waves, the coordination between the body undulations and the limbs, and the mechanisms of gait transition.

The new robot will allow us to study new hypotheses, in particular related to the important role of sensory feedback in 


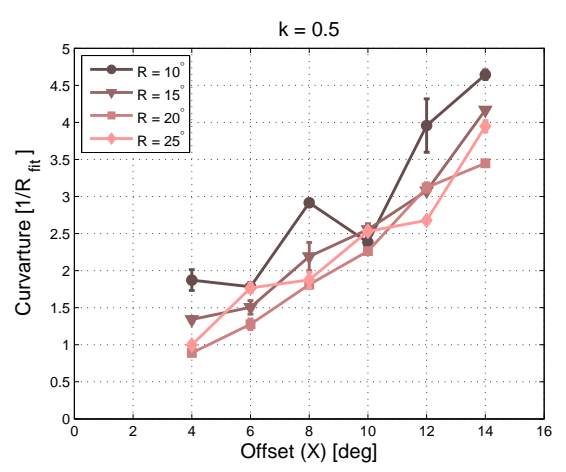

(a)

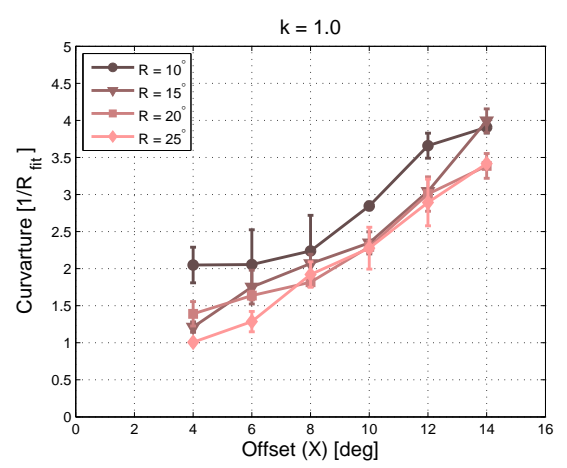

(b)

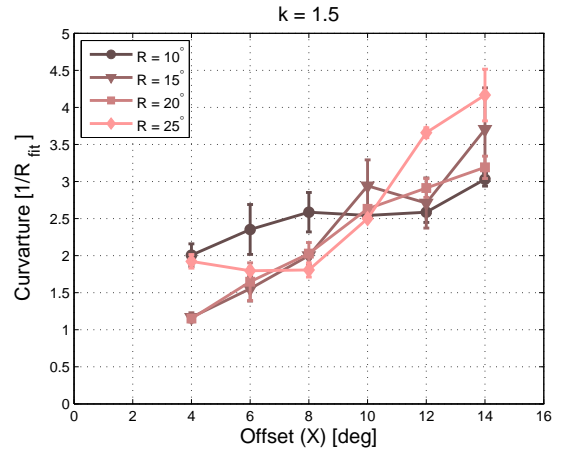

(c)

Fig. 11. Experiment E. Curvature of turning during swimming for (a) $k=0.5$, (b) $k=1.0$ and (c) $k=1.5$, for different amplitudes ( $R$ ) and offsets $(X)$ of the body's oscillation. The error bars correspond to the standard deviation of the three estimations of the curvature at different sites of the resulting trajectory.

gait transition (forthcoming article). We also plan to include a muscle model between the CPG output and the low-level motor controller.

Improvements of the robot compared to the previous one are faster locomotion and a better match to salamander kinematics. In particular the swimming speed of Salamandra robotica I was significantly slower than the real salamander, and we had hypothesized in [6] that this was due to a combination of less profiled limbs, low torque limits, and fewer DOFs compared to the real animal.

\section{A. Future work}

The robot can still be improved. One of the main limitations of the current prototype is that it performs essentially planar locomotion. Because the spine cannot actively bend in the vertical plane the robot has only very limited capabilities to go over obstacles. Also the limbs with only one DOF are too simple. This prevents any application of the current robot in outdoor environments, e.g., for search-and-rescue scenarios or inspection tasks. The use of the robot for real-world applications would also need improvements of the robot's controller, for example to automatically adapt the gait and its parameters to the environment.

The energy consumption measurement capabilities of the current robot are quite limited. A more precise measurement system would allow us, for example, to compare the energy efficiency of the different gaits.

A robot with more DOFs in the limbs is therefore under construction. To go towards real applications we are also working on adding more sensory capabilities to the robot: implementing a binocular camera system, water detection sensors along the spine and an inertial measurement unit.

Concerning using the robot to study animal locomotion, we intend to make several studies in the near future: exploration of role of sensory feedback in pattern generation (as mentioned above), study of energy consumption and cost of travel (compare optima for speed and for efficiency) and study the richness of salamander motor behavior (i.e., other behaviors such as backwards walking, aquatic walking, paddling movement, scratching movements, etc.).

\section{B. Conclusion}

We strongly believe that robots will be increasingly used in neuroscience to test hypotheses concerning sensorimotor loops. These studies require realistic "embodiments" and interactions with the environment. Robots can play a significant role in providing embodiments that are difficult to simulate numerically using physics-based simulators, e.g., interaction forces with a complex terrain or the hydrodynamics of anguilliform swimming (the hydrodynamics around a deformable body are notoriously hard to simulate properly). A large challenge is therefore to design robots that come as close as possible to perform the motor behaviors exhibited by animals. This article contributed a hopefully significant step in that direction.

\section{ACKNOWLEDGMENT}

This work has been supported by the Swiss National Science Foundation, and by the European Community's FP7 projects LAMPETRA and ANGELS.

\section{REFERENCES}

[1] A.H. Cohen. Evolution of the vertebrate central pattern generator for locomotion. In A. H. Cohen, S. Rossignol, and S. Grillner, editors, Neural Control of Rhythmic Movements in Vertebrates. Jon Wiley \& Sons, 1988.

[2] K.-Q. Gao and N.H. Shubin. Late jurassic salamanders from northern China. Nature, 410:574-577, 2001.

[3] G. Roth, K. Nishikawa, C. Naujoks-Manteuffel, A. Schmidt, and D.B. Wake. Paedomorphosis and simplification in the nervous system of salamanders. Brain, Behavior and Evolution, 42:137-170, 1993.

[4] G. Roth, K. Nishikawa, and D.B. Wake. Genome size, secondary simplification, and the evolution of the brain in salamanders. Brain, Behavior and Evolution, 50:50-59, 1997.

[5] A.J. Ijspeert. A connectionist central pattern generator for the aquatic and terrestrial gaits of a simulated salamander. Biological Cybernetics, 84(5):331-348, 2001.

[6] A.J. Ijspeert, A. Crespi, D. Ryczko, and J.-M. Cabelguen. From swimming to walking with a salamander robot driven by a spinal cord model. Science, 315(5817):1416-1420, 2007.

[7] B. Webb. What does robotics offer animal behaviour? Animal Behaviour, 60:545-558, 2000.

[8] C. Wilbur, W. Vorus, Y. Cao, and S.N. Currie. Neurotechnology for biomimetic robots, chapter A Lamprey-Based Undulatory Vehicle. Bradford/MIT Press, 2002.

[9] C. Stefanini, G. Orlandi, A. Menciassi, Y. Ravier, G. La Spina, S. Grillner, and P. Dario. A mechanism for biomimetic actuation in lamprey-like robots. In Proceedings of the First IEEE/RAS-EMBS International Conference on Biomedical Robotics and Biomechatronics (BioRob 2006), pages 579-584, 2006. 
[10] J. Ayers and J. Crisman. The lobster as a model for an omnidirectional robotic ambulation control architecture. In R.D. Beer, R.E. Ritzmann, and T.M. McKenna, editors, Biological neural networks in invertebrate neuroethology and robotics, pages 287-316. Academic Press, 1993.

[11] B. Webb and R. Reeve. Reafferent or redundant: Integration of phonotaxis and optomotor behavior in crickets and robots. Adaptive Behavior, 11(3):137-158, 2003.

[12] Y. Fukuoka, H. Kimura, and A.H. Cohen. Adaptive dynamic walking of a quadruped robot on irregular terrain based on biological concepts. The International Journal of Robotics Research, 22(3-4):187-202, 2003.

[13] B. Webb. Can robots make good models of biological behaviour? Behavioral and brain sciences, 24:1033-1050, 2001.

[14] B. Webb. Robots in invertebrate neuroscience. Nature, 417:359-363, 2002.

[15] R. Breithaupt, J. Dahnke, K. Zahedi, J. Hertzberg, and F. Pasemann. Robo-salamander - an approach for the benefit of both robotics and biology. In Proceedings of the $5^{\text {th }}$ International Conference on Climbing and Walking Robots (CLAWAR 2002), 2002.

[16] A. Hiraoka and H. Kimura. A development of a salamander robot design of a coupled neuro-musculoskeletal system. In Proceedings of the Annual Conference of the Robotics Society of Japan, Osaka, 2002. (Paper in Japanese).

[17] K. Sangbae, M. Spenko, S. Trujillo, B. Heyneman, D. Santos, and M.R. Cutkosky. Smooth vertical surface climbing with directional adhesion. IEEE Transactions on Robotics, 24(1):65-74, 2008.

[18] M. Sfakiotakis and D.P. Tsakiris. Pedundulatory robotic locomotion: Centipede and polychaete modes in unstructured substrates. In Proceedings of the 2008 IEEE International Conference on Robotics and Biomimetics, pages 651-658, 2008.

[19] J. Ayers, C. Wilbur, and C. Olcott. Lamprey robots. In T. Wu and N. Kato, editors, Proceedings of the International Symposium on Aqua Biomechanisms, 2000.

[20] P. Arena. A mechatronic lamprey controlled by analog circuits. In Proceedings of the $9^{\text {th }}$ IEEE Mediterranean Conference on Control and Automation (MED '01), 2001.

[21] A.J. Ijspeert and A. Crespi. Online trajectory generation in an amphibious snake robot using a lamprey-like central pattern generator model. In Proceedings of the 2007 IEEE International Conference on Robotics and Automation (ICRA 2007), pages 262-268, 2007.

[22] A. Crespi and A.J. Ijspeert. Online optimization of swimming and crawling in an amphibious snake robot. IEEE Transactions on Robotics, 24(1):75-87, 2008.

[23] C. Stefanini, S. Orofino, L. Manfredi, S. Mintchev, S. Marrazza, T. Assaf, L. Capantini, E. Sinibaldi, S. Grillner, P. Wallén, and P. Dario. A novel autonomous, bioinspired swimming robot developed by neuroscientists and bioengineers. Bioinspiration and Biomimetics. In press.

[24] J. Ayers, J. Witting, N. McGruer, C. Olcott, and D. Massa. Lobster robots. In T. Wu and N. Kato, editors, Proceedings of the International Symposium on Aqua Biomechanisms, 2000.

[25] G. Dudek, P. Giguere, C. Prahacs, S. Saunderson, J. Sattar, L.-A. TorresMendez, M. Jenkin, A. German, A. Hogue, A. Ripsman, J. Zacher, E. Milios, H. Liu, P. Zhang, M. Buehler, and C. Georgiades. AQUA: An amphibious autonomous robot. Computer, 40(1):46-53, 2007.

[26] L.M. Frolich and A.A. Biewener. Kinematic and electromyographic analysis of the functional role of the body axis during terrestrial and aquatic locomotion in the salamander Ambystoma tigrinum. Journal of Experimental Biology, 62:107-130, 1992.

[27] I. Delvolvé, T. Bem, and J.-M. Cabelguen. Epaxial and limb muscle activity during swimming and terrestrial stepping in the adult newt, Pleurodeles waltl. Journal of Neurophysiology, 78:638-650, 1997.

[28] R. Bainbridge. Caudal fin and body movement in the propulsion of some fish. Journal of Experimental Biology, 40(1):23-56, 1963.

[29] K. D'Août and P. Aerts. Kinematics and efficiency of steady swimming in adult axolotls (Ambystoma mexicanum). Journal of Experimental Biology, 200:1863-1871, 1997.

[30] M.A. Ashley-Ross. Metamorphic and speed effects on hindlimb kinematics during terrestrial locomotion in the salamander Dicamptodon tenebrosus. Journal of Experimental Biology, 193:285-305, 1994.

[31] M. Hildebrand. Neural Control of Locomotion, chapter Analysis of tetrapod gaits: general considerations and symmetrical gaits, pages 203 236. Plenum Press, 1976.

[32] S. Daan and T. Belterman. Lateral bending in locomotion of some lower tetrapods. Proceedings of the Koninklijke Nederlandse Akademie van Wetenschappen. Series C, 71:245-266, 1968.

[33] P. Roos. Lateral bending in newt locomotion. Proceedings of the Koninklijke Nederlandse Akademie van Wetenschappen. Series C, 67:223-232, 1964.
[34] D.R. Carrier. Action of the hypaxial muscles during walking and swimming in the salamander Dicamptodon ensatus. Journal of Experimental Biology, 180:75-63, 1993.

[35] W. Bennett, R. Simons, and E. Brainerd. Twisting and bending: the functional role of salamander lateral hypaxial musculature during locomotion. Journal of Experimental Biology, 204:1979-1989, 2001.

[36] S. Chevallier, A.J. Ijspeert, D. Ryczko, F. Nagy, and J.-M. Cabelguen. Organisation of the spinal central pattern generators for locomotion in the salamander: biology and modelling. Brain Research Reviews, 57(1):147-161, 2008.

[37] O.R. Barclay. The mechanics of amphibian locomotion. Journal of experimental biology, 23:177-203, 1946.

[38] N. Harischandra, J.-M. Cabelguen, and Ö. Ekeberg. A 3D musculomechanical model of the salamander for the study of different gaits and modes of locomotion. Frontiers in Neurorobotics, 4, 2010.

[39] E. Azizi and T. Landberg. Effects of metamorphosis on the aquatic escape response of the two-lined salamander (Eurycea bislineata). Journal of Experimental Biology, 205(6):841-849, 2002.

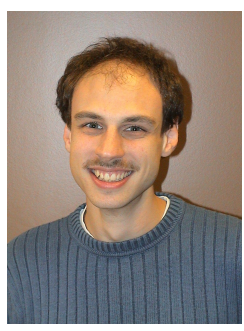

Alessandro Crespi is a postdoctoral researcher at the Biorobotics Laboratory at EPFL. He has a $\mathrm{BSc} / \mathrm{MSc}$ and $\mathrm{PhD}$ in computer science from the EPFL. His research interests are in the field of biologically inspired amphibious robots. He is mainly working on the development of the electronics of the robots, and on the experiments to characterize their locomotion.

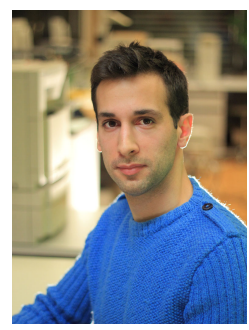

Konstantinos Karakasiliotis is a $\mathrm{PhD}$ student at the Biorobotics Laboratory at EPFL. He has a BSc/MSc in Electronics and Computer Engineering at the Technical University of Crete - Greece (T.U.C.) His research interests are in the field of bioinspired robotics and, more precisely, in the design and locomotion control of bioinspired robots.

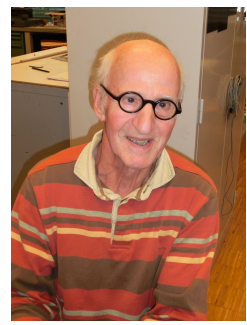

André Guignard is retired since March 2007 but still collaborates with BioRob and other EPFL laboratories. After a formation as watchmaker, he became engineer. He was enrolled at the EPFL in 1976. Working with Prof. J.-D. Nicoud, he designed and built in 1977 the first modern computer mouse. $\mathrm{He}$ is the creator of the mechanics of the Khepera, of the S-Bot and of several other mobile robots.

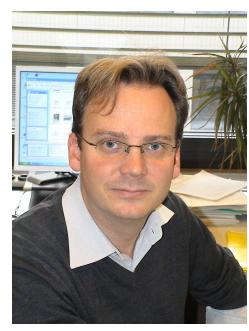

Auke Jan Ijspeert is professor at the EPFL (the Swiss Federal Institute of Technology at Lausanne), and head of the Biorobotics Laboratory. He has a $\mathrm{BSc} / \mathrm{MSc}$ in physics from the EPFL, and a PhD in artificial intelligence from the University of Edinburgh. His research interests are at the intersection between robotics, computational neuroscience, nonlinear dynamical systems, and applied machine learning. He is interested in using numerical simulations and robots to get a better understanding of sensorimotor coordination in animals, and in using inspiration from biology to design novel types of robots and adaptive controllers. With his colleagues, he has received the Best Paper Award at ICRA2002, the Industrial Robot Highly Commended Award at CLAWAR2005, and the Best Paper Award at the IEEE-RAS Humanoids 2007 conference. He was the Technical Program Chair of 5 international conferences (BioADIT2004, SAB2004, AMAM2005, BioADIT2006, LATSIS2006), and has been a program committee member of over 40 conferences. 